\title{
Activation of interferon signaling pathway in primary effusion lymphoma cells by inhibition of viral interferon regulatory factor I of Kaposi's sarcoma-associated herpesvirus Y Zhang*, D Patel and S Fan
}

Address: Virginia-Maryland Regional College of Veterinary Medicine and Maryland Pathogen Research Institute, University of Maryland, College Park, Maryland, USA

* Corresponding author

from I I th International Conference on Malignancies in AIDS and Other Acquired Immunodeficiencies (ICMAOI): Basic, Epidemiologic, and Clinical Research

Bethesda, MD, USA. 6-7 October 2008

Published: 17 June 2009

Infectious Agents and Cancer 2009, 4(Suppl 2):P47 doi:I0.II86/I750-9378-4-S2-P47

This abstract is available from: http://www.infectagentscancer.com/content/4/S2/P47

(C) 2009 Zhang et al; licensee BioMed Central Ltd.

Kaposi's Sarcoma-Associated Herpesvirus (KSHV), also known as human Herpesvirus 8, is associated with several malignant disorders, including Kaposi's sarcoma, primary effusion lymphoma (PEL) and multicentric Castleman's disease. KSHV ORF-K9 encodes viral interferon regulatory factor 1 (vIRF1), an early lytic gene product homologous to cellular interferon regulatory factors. The IRF family members are transcription regulatory factors that bind to interferon-stimulated response elements (ISRE) to regulate interferon-responsive genes involved in pathogen response, cytokine signaling, cell growth regulation, and hematopoietic development. As an oncogenic protein, VIRF1 potentially contributes to KSHV-driven malignancies. In this study, KSHV vIRF1 was suppressed with peptide-conjugated antisense phosphorodiamidate morpholino oligonucleotides (PPMO) and the effect was examined. PPMO are single-stranded DNA analogues that have a modified backbone and which penetrate cells readily. Treatment of PEL cells with anti-K9 PPMO led to reduction of vIRF1 expression. The anti-K9 PPMO also reduced expression of vIRF1 in 293 cells transiently transfected with a K9 plasmid. PEL cells that were treated with anti-K9 PPMO exhibited reduced RTA and vIL- 6 expression and reduced level of KSHV DNA copies. The inhibition of VIRF1 in PEL cells led to elevation of protein levels of important genes in IFN signaling, including IRF3 and STAT1, indicating the activation of the pathway. The results demonstrate that targeting of vIRF1 mRNA with PPMO can potently reduce vIRF1 protein translation, and indicate that further exploration of these compounds in an animal model is warranted. 